\title{
Prevalence and determinants of modern contraceptive utilization among rural lactating mothers: Findings from the primary health care project in two northwest Ethiopian districts
}

Terefe Derso ( $\square$ dersotere@gmail.com )

University of Gondar

Gashaw Andargie Biks

University of Gondar

Mezgebu Yitayal

University of Gondar

Tadesse Awoke Ayele

University of Gondar

Kassahun Alemu

University of Gondar

Getu Debalkie

University of Gondar

Hailab Fekadu

University of Gondar

Endalkachew Dellie

University of Gondar

Telake Azale

University of Gondar

Bisrat Misganaw

University of Gondar

Adane Kebede

University of Gondar

Destaw Fetene

University of Gondar

Tsegaye G/medhin

University of Gondar

Asmamaw Atnafu

University of Gondar 
Research article

Keywords: Contraceptive utilization, postnatal care, Child immunization

Posted Date: March 20th, 2020

DOI: https://doi.org/10.21203/rs.2.15782/v3

License: (c) (i) This work is licensed under a Creative Commons Attribution 4.0 International License. Read Full License

Version of Record: A version of this preprint was published at BMC Women's Health on April 3rd, 2020. See the published version at https://doi.org/10.1186/s12905-020-00933-7. 


\section{Abstract}

Background: Contraceptive utilization is a guarantee to avert unwanted pregnancies. In Ethiopia however, more than half of the rural women have shorter birth intervals. Consequently, $17 \%$ and $8 \%$ of the births have been either mistimed (wanted at later date) or unwanted, respectively. Therefore, this study investigated modern contraceptive utilization and its predictors among rural lactating women. Methods: A community based-cross-sectional study was conducted from May 01 to June 29, 2019, in Dabat and Gondar zuria districts, northwest Ethiopia. Data from 603 lactating mother were collected through face to face interviews using a structured questionnaire. Bivariate and multivariate logistic regression analyses were fitted to identify the independent predictors of modern contraceptive utilization. Results: The overall prevalence of modern contraceptive (MC) utilization rate was $45.8 \%$ [95\% Cl: 38.01, 53.59]. The contraceptive method mix was dominated by Depo-Provera (39.8\%) followed by implants (4.8\%). The odds of utilization of contraceptive were 5.58 times higher among mothers of children with fully immunized $[A O R=5.58,95 \% \mathrm{Cl}: 3.45,9.01]$ compared to mothers whose children were vaccinated partially or not at all. Mothers who received antenatal [AOR=1.74, 95\% Cl: $1.13,4.43]$ and postnatal care [AOR=2.02, 95\% Cl: $1.24,2.91$ ) were 1.74 and 2.02 folds more likely to utilize modern contraceptives than mothers who did not receive such care, respectively. Conclusion: The prevalence of modern contraceptive utilization in this study area was lower than the planed national target. In the region, child immunization service is one of the promising platforms for reaching lactating mothers with modern contraceptive utilization. Our findings suggest that antenatal and postnatal care visits are the other key determinants of modern contraceptive utilization. Thus, in low-resource settings like ours, the health system approaches to improved antenatal and, postnatal care and child immunization services should be intensified with more effective advice on modern contraceptive utilization to reduce unwanted pregnancies. Key words: Contraceptive utilization, postnatal care, Child immunization

\section{Background}

The health benefits of contraceptive use include preventing unplanned pregnancies [1] and averting maternal and child morbidity by allowing couples to space their pregnancies by more than two years [2, 3 , 4]. In spite of this, more than half of the lactating women are at high risk for unwanted or unplanned pregnancy immediately after birth [5]. The postpartum period, the time of care for themselves and their newborns provides an important opportunity for contacting the health system [6]. In fact, postpartum modern contraceptive has the potential of reduce $71 \%$ of unwanted and abolishing 53 million of the unintended pregnancies as well as resulting in 22, 25 and 7 million fewer unplanned births, induced abortion and miscarriages, respectively [7]. Also, longer birth intervals or contraceptive uses can avert about 1 in 5 deaths in children of 1 to 4 years of age [8] and $44.3 \%$ of maternal deaths [9].

Since 1993 Ethiopia has been implementing a clear population policy [10], and one of the major strategies of the policy currently the family planning program to raise contraceptive prevalence to $55 \%$ by 2020 through free of charge provisions at both government and non-government health facilities $[11,12]$. So far, modern contraceptive prevalence is low (35\%) with significant regional variations, like for example, 
Somali (1\%), Affar (12\%) and Amhara [47\%]. Consequently, $17 \%$ and $8 \%$ of the births were mistimed (wanted at later date) and unwanted, respectively [13].

Contraceptive uptake has been positively associated with a range of both health and non-health related outcomes. Factors that influence contraceptive practice are multifaceted and complex. Previous studies shown that socio-cultural, health and socio-economic characteristics are main significant determinants of modern contraceptive utilization. High contraceptive utilization has been documented among literate [13, 14], old age [14], rural residences [13], media familiar and richest women [15] who have had many (>5) live children $[16,17]$. Health care utilizations during pregnancy and after delivery, antenatal and postnatal care $[18,19]$ and child immunization [20] also increase the likelihood of modern contraceptive utilization.

Mothers wish to space their births sufficiently far apart that even when their children a toddlers, they prefer to delay pregnancies [21]. However, in Ethiopia more than half of the rural women have shorter birth intervals; consequently, high maternal mortality rate is documented [13]. Also subsequent conceptions and births negatively affect mothers' decisions to continue breast-feeding children [22]. Some local studies have focused on assessing the contraceptive utilization status of reproductive women generally $[16,17]$, only two local studies in Ethiopia targeted to urban lactating women [23, 24]. Thus, the lactating period is a window of opportunity to improve contraceptive utilization. Investigating the prevalence and predictors of modern contraceptive utilization in rural area is of a vital importance to design strategies to address the problem. Therefore, this study investigated modern contraceptive utilization and its predictors among rural lactating women.

\section{Methods}

\section{Study setting and design}

A community-based cross-sectional study was conducted from May 01 to June 29, 2019 in Dabat and Gondar Zuria districts, northwest Ethiopia. Two of the total 23 districts in North Gondar zone of the Amhara region, Dabat and Gondar zuria districts, consist of 30 and 38 kebeles(the smallest administrative units in Ethiopia), respectively. Located in different ecological zones (high, middle, and low land), the districts had 145,509(Dabat) and 231,324(Gondar zuria ) inhabitants who's largely depended on subsistence farming. Of the total inhabitants, 5973 in Dabat and 8,180 in Gondar zuria district were lactating mothers, respectively.

\section{Study population and sampling procedure}

Lactating mothers who lived in Dabat and Gondar zuria districts for at least six months were included. The study aimed to assess the prevalence and determinants of modern contraceptive utilization among rural lactating mothers in Dabat and Gondar zuria districts, northwest Ethiopia. Of the total kebeles, eight in Dabat and ten in Gondar zuria were selected using the lottery method. The systematic sampling technique was used to select study participants. For households with multiple lactating mothers who fulfilled the inclusion criteria, the lottery method was used to choose one. 
Sample size was calculated using Epi-info version 3.7 by considering the assumptions: $48.4 \%$ prevalence of modern contraceptive utilization in Gondar town [23], $95 \%$ level of confidence and $5 \%$ margin of error. A design effect of 1.5 and $10 \%$ non-response rate were also anticipated to obtain the final sample size of 631.

\section{Data collection tools and procedure}

A structured pre-tested questionnaire was designed to capture socio-demographic characteristics and primary health care utilization (immunization, family planning, health education, hygiene and sanitation, maternal health service and physical access to health services). The questionnaire was first prepared in English and translated to Amharic (the native language of the study area). Back translation to English was made to compare the consistency and amendments accordingly. Fifteen data collectors and three field supervisors were recruited for data collection. Two days training was given on the objective of the study, confidentiality of information and the techniques of an interview to data collectors and supervisors.

\section{Study variables and data analysis}

To ascertain the outcome variable, modern utilization of contraceptive, women were asked if they were currently using a method of contraception and what method they were using. The outcome was coded 1 if they were using a modern method (pill, Intra uterine contraceptive device (IUCD), injections, condom, male or female sterilization, implant, or diaphragm/foam/jelly) and 0 if they were using a traditional method or were not currently using a method. Epi-data version 3.1 was used for data entry and data were exported to SPSS version 21 for analysis. Descriptive statistics were computed. Binary Logistic regression model was used to identify the relationship between dependent and independent variables. Those significant independent variables in bivariate analysis ( $p$-value $<0.2)$ were entered into the multivariable analysis. In the binary logistic regression model, backward- stepwise multivariate analysis was used to elicit associated factors of modern utilization of contraceptive. In the final model, a significant association was declared at a p-value less than 0.05 and finally, the results were presented in texts and tables with adjusted odds ratio (AOR) and the corresponding 95\% confidence interval.

\section{Results}

\section{Socio-demographic characteristics of study participants}

A total of 603 mothers (with a response rate of $95.56 \%$ ) participated in the study. Most of mothers $(94.7 \%)$ were housewives. Only nearly one-fourth $(22.1 \%)$ had formal education; $22.9 \%$ were in the age ranges of 36-49 years (Table 1 ).

Table 1: Socio-demographic characteristics of study participants in the rural population of northwest Ethiopia 


\begin{tabular}{|l|l|l|}
\hline Characteristics & Frequency & Percent \\
\hline Age of mothers(in years) & & \\
\hline $18-24$ & 102 & 16.9 \\
\hline $25-35$ & 363 & 60.2 \\
\hline $36-49$ & 138 & 22.9 \\
\hline Maternal educational status & & \\
\hline Unable to read and write & 400 & 66.3 \\
\hline Able to read and write without formal education & 70 & 11.6 \\
\hline Formal education & 133 & 22.1 \\
\hline Husband's educational status & & \\
\hline Unable to read and write & 344 & 57.0 \\
\hline Able to read and write without formal education & 133 & 22.1 \\
\hline First cycle(1-4 grade) & 41 & 6.8 \\
\hline Second cycle(5-8 grade) & 50 & 8.3 \\
\hline Secondary school(9-12 grade) & 26 & 4.3 \\
\hline Certificate and above & 9 & 1.5 \\
\hline Occupation of mothers & & \\
\hline House wife & 571 & 94.7 \\
\hline Outdoor worker & 32 & 5.3 \\
\hline Number of children & 181 & 30.0 \\
\hline $1-4$ & & \\
\hline Above 4 children & & \\
\hline
\end{tabular}

\section{Health related characteristics of study participants}

Of all participants, $95.2 \%$ had antenatal care (ANC) visits; $70.0 \%$ delivered in health institutions (Table 2).

Table 2: Health related characteristics of study participants in the rural population of northwest, Ethiopia 


\begin{tabular}{|l|l|l|}
\hline Characteristics & Frequency & Percent \\
\hline Travel time to the nearest health center(HC) & & \\
\hline 30 or below minutes & 146 & 24.2 \\
\hline Above 30 minutes & 457 & 75.8 \\
\hline ANC visit during your last pregnancy & & \\
\hline Yes & 574 & 95.2 \\
\hline No & 29 & 4.8 \\
\hline Place of last delivery & & \\
\hline Home & 181 & 30.0 \\
\hline Health institution & 422 & 70.0 \\
\hline Postnatal care in the current delivery & & \\
\hline Yes & 397 & 65.8 \\
\hline No & 206 & 34.2 \\
\hline Place of postnatal care & & \\
\hline Hospital & 22 & 3.6 \\
\hline Health Center & 267 & 44.3 \\
\hline Health Post & 108 & 17.9 \\
\hline Child immunization status & & \\
\hline Fully vaccinated & 1394 & 23.1 \\
\hline Partially/not vaccinated & \\
\hline
\end{tabular}

\section{Prevalence of modern contraceptive utilization}

The prevalence of modern contraceptive (MC) utilization was $45.8 \%$ [95\% Cl: 38.01, 53.59]. The contraceptive method mix was dominated by Depo-Provera (39.8\%) followed by implants (4.8\%). However, very few mothers (1.2\% and $0.2 \%$ ) utilized pills and Intra uterine contraceptive devices, respectively.

\section{Factors associated with modern contraceptive utilization}

In the bivariable analysis age of mothers, number of children, place of last delivery, travel time to the nearest health center, antenatal care visit, postnatal care and child immunization status were found associated with a p-value of less than 0.2 . However, the result of the multivariable analysis revealed with a p-value of less than 0.05 that the odds of modern utilization of contraceptive were 5.58 times higher among with children with fully immunization [AOR $=5.58,95 \% \mathrm{Cl}: 3.45,9.01]$ compared to mothers with children with incomplete or not vaccinated. Mothers who received antenatal care [AOR $=1.74,95 \% \mathrm{Cl}$ : $1.13,4.43]$ and postnatal care[AOR=2.02, 95\% Cl: 1.24, 2.91) were 1.74 and 2.02 folds more likely to utilize 
modern contraceptive than mothers who did not received antenatal care and postnatal care, respectively (Table 3).

Table 3: Factors associated with modern utilization of contraceptive in the rural population of northwest, Ethiopia

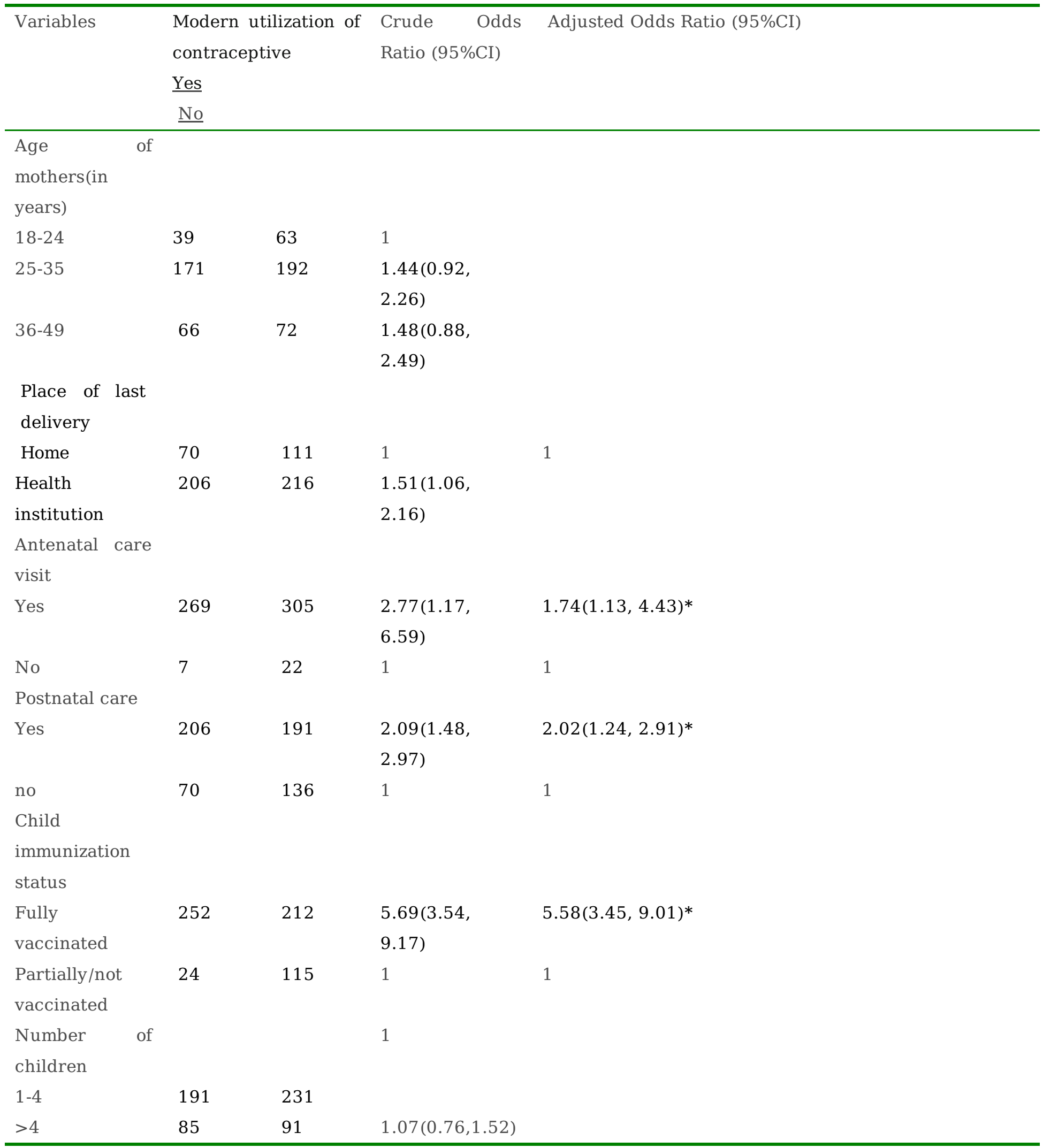


Contraceptives utilization is a guarantee to avert unwanted pregnancies [1]. This community based crosssectional study revealed that the overall prevalence of modern contraceptive utilization was $45.8 \%$ [ $95 \%$ Cl: 38.01, 53.59]; antenatal and postnatal care; and child immunization status were the predictors of the utilization of contraceptives. In this study the magnitude of modern contraceptive utilization was consistent with that of a previous study reported from Gondar city (48.4\%)[23].

However, the magnitude of modern contraceptive utilization in our study is lower than the finding in Malawi where three-fourths (75\%) of the lactating women utilized contraceptives [25]. This difference might be due to variations in the awareness of the people, the availability of the contraceptive methods and differences in study settings to access the service. The high prevalence in Malawi compared to ours might be due to differences the education levels. In our study more than three-fourths $(77.9 \%)$ of the participants were illiterate, while in Malawi, the great majority $(90.2 \%)$ of the participants had primary and above education. Since education enables mothers to have knowledge and awareness about the benefits of family planning, it enhances their modern contraceptive utilization. This implies that educated women have greater ability to stick to health care inputs, such as modern contraceptive utilization which improves birth spacing.

Mothers who received antenatal care were 1.74 folds more likely to utilize modern contraceptive than mothers who did not received such care. These higher odds of modern contraceptive utilization are supported by findings reported from Ethiopia [19] and Nigeria [18]. The plausible reason might be that during antenatal care follow ups the mothers obtain counseling on birth spacing and family planning. Thus, antenatal care is important to reach women with information about the return of fertility, their options to space or limit future pregnancies, and the benefits to their own and their newborn's health of doing so before they are at risk for unintended pregnancies. Recommending that women be counseled on birth spacing and family planning during ANC contacts is worth while

In the multivariate analysis, postnatal care was the other significant variable because mothers who received postnatal care were 2.02 times more likely to utilize modern contraceptive than their counterparts as repeated by other studies in Africa $[19,18]$. This might be so because postnatal care enabled health workers to counsel women on the importance of birth spacing. Therefore, postnatal care should be prioritized with more effective advice on family planning to reduce unintended births.

The uncommon finding of this study showed that the likelihood of modern contraceptive utilization was 5.58 times higher among lactating mothers with fully immunized children compared to mothers of who were either partially immunized or not vaccinated at all. In fact, the recommended vaccination schedule for children allows for multiple health care contacts with infants and their mothers during the first year of life [20]. These routine immunization services offer important opportunities to discuss family planning and to reach many postpartum women during group education talks. In Ethiopia, immunization contacts occur regularly according to the national schedule of $6,10,14$ weeks and 9 and 15 months [26]. Thus, child immunization services are promising Platforms to reach lactating mothers with modern 
contraceptive utilization [27]. Finally, advocacy efforts can promote positive partnerships between child health and family planning programs as a 'win-win' for the health of both the mother and the infant.

Limitation: This study provides important insights about modern contraceptive utilization and its predictors among lactating mothers in Dabat and Gondar Zuria districts, where there is a scarcity of literature. However, the cross sectional nature of the study has limited its capacity of measuring the cause and effect relationships of the outcome and potential predictors.

\section{Conclusions}

In the study area, the prevalence of modern contraceptive utilization was lower documented compared to the planed national figure. In the region, child immunization services were promising platforms to reach lactating mothers with modern contraceptives. Also our findings suggest that antenatal and postnatal care visits are the other key determinants of modern contraceptive utilization. In low-resource settings like Ethiopia, the health system approaches to improved antenatal, postnatal care and child immunization services should be intensified with more effective advice on modern contraceptive utilization to reduce unwanted pregnancies.

\section{List Of Abbreviations}

AOR: Adjusted Odds Ratio; ANC: Antenatal Care; COR: Crude Odds Ratio, Cl: Confidence Interval; IUCD; Intra Uterine Contraceptive Device: PNC: Postnatal Care; SPSS: Statistical Package for Social Science; WHO: World Health Organization

\section{Declarations}

\section{Ethics approval and consent to participate}

Ethical approval was obtained from Institutional Review Board of University of Gondar. Informed consent was also obtained from study participants. The confidentiality of information was guaranteed by using code numbers rather than personal identifiers and by keeping the data locked. Participants were told to decline at any time if they felt uncomfortable, even after the interview was started.

\section{Consent for publication}

Not applicable

\section{Availability of data and materials}

We confirm that all data underlying the findings would be fully available without restriction if the manuscript is published.

Competing interest 
Authors declare that they have no conflict of interest

\section{Funding}

This study was funded by the University of Gondar. The views presented in the article are of the author and not necessarily express the views of the funding organization. The University of Gondar was not involved in the design of the study, data collection, analysis and interpretation.

\section{Authors contributions}

AA TD GAB MY TAA conceived the study, developed the tool, coordinated data collection, and carried out the statistical analysis and drafted the manuscript. AA KA GD HF TA BM AK DF TG AA ED conceived the study, participated in the statistical analysis, and drafted the manuscript. TD AA conceived the study and reviewed the drafted manuscript. All authors read and approved the final manuscript.

\section{Acknowledgements}

We would like to thank the mothers for their willingness to participate in the study. Our appreciation will also go to the University of Gondar for their financial and material support.

\section{References}

1. Sedgh G, Singh S, Hussain R. Intended and unintended pregnancies worldwide in 2012 and recent trends. Studies in family planning. $2014 ; 45(3): 301-14$.

2. DaVanzo J, Hale L, Razzaque A, Rahman M. The effects of pregnancy spacing on infant and child mortality in Matlab, Bangladesh: how they vary by the type of pregnancy outcome that began the interval. Population studies. 2008 ;62(2):131-54.

3. Chen, M.J., Kim, C.R., Whitehouse, K.C., Berry-Bibee, E. and Gaffield, M.E. Development, updates, and future directions of the World Health Organization selected practice recommendations for contraceptive use. International journal of gynaecology and obstetrics: the official organ of the International Federation of Gynaecology and Obstetrics. 2017; 136(2):113.

4. Singh S, Bankole A, Darroch JE. The impact of contraceptive use and abortion on fertility in subSaharan Africa: estimates for 2003-2014. Population and development review. $2017 ; 43(1): 141$.

5. ohannes S, Wondafrash M, Abera M, Girma E. Duration and determinants of birth interval among women of child bearing age in Southern Ethiopia. BMC pregnancy and childbirth. $2011 ; 11$ (1):38.

6. Sines E, Syed U, Wall S, Worley H. Postnatal care: A critical opportunity to save mothers and newborns. Policy Perspectives on Newborn Health. 2007 :1-7.

7. World Health Organization. Packages of interventions for family planning, safe abortion care, maternal, newborn and child health. WHO; 2010.

8. Central Statistical Authority [Ethiopia] and ORC Macro. Mini Ethiopia Demographic and Health Survey 2014. Addis Ababa. Maryland: Ethiopia and Calverton; 2014. 
9. Ahmed S, Li Q, Liu L, Tsui AO. Maternal deaths averted by contraceptive use: an analysis of 172 countries. The Lancet. 2012 ;380(9837):111-25.

10. Federal Democratic Republic of Ethiopia The National Population Policy of Ethiopia; 1993.

11. Federal Democratic Republic of Ethiopia, Ministry of Health. National Guideline for Family Planning Services in Ethiopia; 2011.

12. Federal Ministry of Health; Costed Implementation Plan for Family Planning in Ethiopia 2015-2020; 2016

13. CSA . International: Ethiopia Demographic and Health Survey 2016. Addis Ababa, Ethiopia and Calverton, Maryland, USA: Central Statistical Agency and ICF International.2016.

14. Gebremariam A, Gebremariam H. Contraceptive use among lactating women in Ganta-Afeshum District, Eastern Tigray, Northern Ethiopia, 2015: a cross sectional study. BMC pregnancy and childbirth. $2017 ; 17(1): 421$.

15. Rutaremwa G, Kabagenyi A, Wandera SO, Jhamba T, Akiror E, Nviiri HL. Predictors of modern contraceptive use during the postpartum period among women in Uganda: a population-based cross sectional study. BMC public health. $2015 ; 15(1): 262$.

16. Mohammed A, Woldeyohannes D, Feleke A, Megabiaw B. Determinants of modern contraceptive utilization among married women of reproductive age group in North Shoa Zone, Amhara Region, Ethiopia. Reproductive health. $2014 ; 11(1): 13$.

17. Alemayehu GA, Fekadu A, Yitayal M, Kebede Y, Abebe SM, Ayele TA, Gizaw Z, Wubeshet M, Muchie KF, Gelagay AA, Azmeraw T. Prevalence and determinants of contraceptive utilization among married women at Dabat Health and Demographic Surveillance System site, northwest Ethiopia. BMC women's health. $2018 ; 18(1): 118$.

18. Ajayi Al, Adeniyi OV, Akpan W. Maternal health care visits as predictors of contraceptive use among childbearing women in a medically underserved state in Nigeria. Journal of Health, Population and Nutrition. $2018 ; 37(1): 19$.

19. Teka TT, Feyissa TR, Melka AS, Bobo FT. Role of antenatal and postnatal care in contraceptive use during postpartum period in western Ethiopia: a cross sectional study. BMC research notes. 2018 ;11(1):581.

20. Wallace A, Dietz V, Cairns KL. Integration of immunization services with other health interventions in the developing world: what works and why? Systematic literature review. Tropical Medicine \& International Health. $2009 ; 14(1): 11-9$.

21. Greenhalgh S, Bongaarts J. Fertility policy in China: future options. Science. 1987 ;235(4793):116772.

22. Jayachandran S. Does contraceptive use always reduce breast-feeding?. Demography. 2014 ;51(3):917-37.

23. Abera Y, Mengesha ZB, Tessema GA. Postpartum contraceptive use in Gondar town, Northwest Ethiopia: a community based cross-sectional study. BMC women's health. $2015 ; 15(1): 19$. 
24. Taye EB, Mekonen DG, Debele TZ. Prevalence of post partum modern family planning utilization and associated factors among postpartum mothers in Debre Tabor town, North West Ethiopia. BMC research notes. $2019 ; 12(1): 430$.

25. Bwazi C, Maluwa A, Chimwaza A, Pindani M. Utilization of postpartum family planning services between six and twelve months of delivery at Ntchisi District Hospital, Malawi. Health. 2014 ;6(14):1724.

26. Federal ministry of health. routine EPI schedule. Mothers and child card. 2018.

27. Huntington $D$, Aplogan $A$. The integration of family planning and childhood immunization services in Togo. Studies in Family Planning. 1994;25(3):176-83. 